Research Article

\title{
Generalized Conformable Mean Value Theorems with Applications to Multivariable Calculus
}

\author{
Francisco Martínez, ${ }^{1}$ Inmaculada Martínez, ${ }^{1}$ Mohammed K. A. Kaabar ${ }^{D},{ }^{2}$ \\ and Silvestre Paredes ${ }^{1}$ \\ ${ }^{1}$ Department of Applied Mathematics and Statistics, Technological University of Cartagena, Cartagena 30203, Spain \\ ${ }^{2}$ Jabalia Camp, UNRWA Palestinian Refugee Camp, Gaza Strip, State of Palestine
}

Correspondence should be addressed to Mohammed K. A. Kaabar; mohammed.kaabar@wsu.edu

Received 21 February 2021; Revised 15 March 2021; Accepted 22 March 2021; Published 2 April 2021

Academic Editor: Ahmet Ocak Akdemir

Copyright (C) 2021 Francisco Martínez et al. This is an open access article distributed under the Creative Commons Attribution License, which permits unrestricted use, distribution, and reproduction in any medium, provided the original work is properly cited.

\begin{abstract}
The conformable derivative and its properties have been recently introduced. In this research work, we propose and prove some new results on the conformable calculus. By using the definitions and results on conformable derivatives of higher order, we generalize the theorems of the mean value which follow the same argument as in the classical calculus. The value of conformable Taylor remainder is obtained through the generalized conformable theorem of the mean value. Finally, we introduce the conformable version of two interesting results of classical multivariable calculus via the conformable formula of finite increments.
\end{abstract}

\section{Introduction}

The history of fractional calculus goes back to the late seventeenth century when L'Hospital proposed the fractional-order derivative. With the introduction of fractional calculus, various newly proposed definitions have been introduced. Some of the common definitions are the Caputo, Riesz, Riesz-Caputo, and Riemann-Liouville fractional ones (refer to $[1,2]$ for more information about fractional definitions, and see $[3,4]$ for research studies on the mathematical analysis of fractional calculus). A new local-type fractional definition [5] of derivative and integral has been recently proposed by Khalil et al. in [6]. Conformable derivative is basically considered as a natural extension of the classical derivative that satisfies the properties of usual derivative. In addition, conformable derivative is a generalized version of q-derivative or fractal derivative (refer to the introduction section in [7] for discussion about this relationship). Almeida et al. (2016) discussed in [8] that conformable derivative is an interesting topic of research that deserves to be studied further. In addition, both Zhao \& Luo (2017) and Khalil et al. (2019) presented the physical and geometrical meaning of conformable derivative in $[9,10]$, respectively. Tuan et al. [11] investigated the mild solutions' existence and regularity of the proposed initial value problem for time diffusion equation in the sense of conformable derivative. This main goal of this newly introduced definition is to overcome the difficulties associated with obtaining the solutions for the equations formulated in the sense of nonlocal fractional definitions [12]. Motivated by the introduction of this definition, several research works have been conducted on the mathematical analysis of functions of a real variable formulated in the sense of conformable definition such as chain rule, mean value theorem, Rolle's theorem, power series expansion, and integration by parts formulas $[6,12-14]$. The conformable partial derivative of the order $\alpha \in(0,1]$ of the real-valued functions of several variables and the conformable gradient vector has been defined as well as the conformable Clairaut's theorem for partial derivative has also been studied in [15]. The conformable Jacobian matrix has been proposed in [16], and the chain rule for multivariable conformable derivative has also been proposed. The conformable Euler's theorem on homogeneous has been successfully defined in [17]. 
Furthermore, many research studies have been conducted on the theoretical and practical elements of conformable differential equations shortly after the proposition of this new definition [5, 7, 12, 18-35]. Conformable derivative has also been applied in modeling and investigating phenomena in applied sciences and engineering [12] such as the nonlinear Boussinesq equation's travelling wave solutions [36], the coupled nonlinear Schrödinger equations [34] and regularized long wave Burgers equation [35] deterministic and stochastics forms, the approximate long water wave equation's exact solutions [37], the $(1+3)$-ZakharovKuznetsov equation with power-law nonlinearity analytical and numerical solutions [38], the $(2+1)$-dimensional Zoomeron equation $[39,40]$ and $3^{\text {rd }}$-order modified $\mathrm{KdV}$ equation analytical solutions [39], and the exact solutions for Whitham-Broer-Kaup equation's three various models in shallow water [41].

The paper is organized as follows: The main concepts of the conformable calculus are presented in the next section. After that, with the help of the definitions and results on conformable derivatives of higher order, the theorems of the mean value are generalized which follow the same argument as in the classical calculus. We also introduce the value of conformable Taylor remainder via the generalized conformable theorems of the mean value. Finally, we characterize the functions of several variables in which one of their conformable partial derivatives is null, and we also obtain the first conformable formula of finite increments.

\section{Basic Definitions and Tools}

Definition 1. Given a function $f:[0, \infty) \longrightarrow R$. Then, the conformable derivative of order $\alpha$ [6] is defined by

$$
\left(T_{\alpha} f\right)(t)=\lim _{\varepsilon \longrightarrow 0} \frac{f\left(t+\varepsilon t^{1-\alpha}\right)-f(t)}{\varepsilon},
$$

for all $t>0,0<\alpha \leq 1$. If $f$ is $\alpha$ differentiable in some $(0, a)$, $a>0$, and $\lim _{t \rightarrow 0^{+}}\left(T_{\alpha} f\right)(t)$ exists, then it is defined as

$$
\left(T_{\alpha} f\right)(0)=\lim _{t \longrightarrow 0^{+}}\left(T_{\alpha} f\right)(t) .
$$

Theorem 1 (see [6]). If a function $f:[0, \infty) \longrightarrow R$ is $\alpha$-differentiable at $t_{0}>0,0<\alpha \leq 1$, then $f$ is continuous at $t_{0}$.

Theorem 2 (see [6]). Let $0<\alpha \leq 1$, and let $f, g$ be $\alpha$ differentiable at a point $t>0$. Then, we have

(i) $T_{\alpha}(a f+b g)=a\left(T_{\alpha} f\right)+b\left(T_{\alpha} g\right), \forall a, b \in R$.

(ii) $T_{\alpha}\left(t^{p}\right)=p t^{p-\alpha}, \forall p \in R$.

(iii) $T_{\alpha}(\lambda)=0$, for all constant functions $f(t)=\lambda$.

(iv) $T_{\alpha}(f g)=f\left(T_{\alpha} g\right)+g\left(T_{\alpha} f\right)$.

(v) $T_{\alpha}(f / g)=\left(g\left(T_{\alpha} f\right)-f\left(T_{\alpha} g\right) / g^{2}\right)$.

(vi) If, in addition, $f$ is differentiable, then $\left(T_{\alpha} f\right)(t)=t^{1-\alpha}(d f / d t)(t)$.

The conformable derivative of certain functions using the above definition is given as follows:
(i) $T_{\alpha}(1)=0$.
(ii) $T_{\alpha}(\sin (a t))=a t^{1-\alpha} \cos (a t)$.
(iii) $T_{\alpha}(\cos (a t))=-a t^{1-\alpha} \sin (a t)$.
(iv) $T_{\alpha}\left(e^{a t}\right)=a e^{a t}, a \in R$.

Definition 2. The (left) conformable derivative starting from $a$ of a given function $f:[a, \infty) \longrightarrow R$ of order $0<\alpha \leq 1$ [13] is defined by

$$
\left(T_{\alpha}^{a} f\right)(t)=\lim _{\varepsilon \longrightarrow 0} \frac{f\left(t+\varepsilon(t-a)^{1-\alpha}\right)-f(t)}{\varepsilon} .
$$

When $a=0$, it is expressed as $\left(T_{\alpha} f\right)(t)$. If $f$ is $\alpha$ differentiable in some $(a, b)$, then the following can be defined as

$$
\left(T_{\alpha}^{a} f\right)(a)=\lim _{t \longrightarrow a^{+}}\left(T_{\alpha}^{a} f\right)(t)
$$

Theorem 3 Chain Rule (see [13]). Assume $f, g:(a, \infty) \longrightarrow R$ be (left) $\alpha$ differentiable functions, where $0<\alpha \leq 1$. By letting $h(t)=f(g(t)), h(t)$ is $\alpha$ differentiable for all $t \neq a$ and $g(t) \neq 0$; therefore, we have the following:

$$
\left(T_{\alpha}^{a} h\right)(t)=\left(T_{\alpha}^{a} f\right)(g(t)) \cdot\left(T_{\alpha}^{a} g\right)(t) \cdot(g(t))^{\alpha-1} .
$$

If $t=a$, then we obtain

$$
\left(T_{\alpha}^{a} h\right)(a)=\lim _{t \longrightarrow a^{+}}\left(T_{\alpha}^{a} f\right)(g(t)) \cdot\left(T_{\alpha}^{a} g\right)(t) \cdot(g(t))^{\alpha-1} .
$$

Theorem 4 Rolle's Theorem (see [6]). Let $a>0, \alpha \in(0,1]$, and $f:[a, b] \longrightarrow R$ be a given function that satisfies

(i)- $f$ is continuous on $[a, b]$.

(ii) $f$ is $\alpha$-differentiable on $(a, b)$.

(iii)- $f(a)=f(b)$.

Then, there exists $c \in(a, b)$ such that $\left(T_{\alpha} f\right)(c)=0$.

Corollary 1 (see [14]). Let $I \subset[o, \infty), \alpha \in(0,1]$, and $f: I \longrightarrow R$ be a given function that satisfies

(i) - $f$ is $\alpha$ differentiable on $I$.

(ii)- $f(a)=f(b)=0$ for certain $a, b \in I$.

Then, there exists $c \in(a, b)$, such that $\left(T_{\alpha} f\right)(c)=0$.

Theorem 5 Mean Value Theorem (see [6]). Let $a>0$, $\alpha \in(0,1]$, and $f:[a, b] \longrightarrow R$ be a given function that satisfies

(i) $f$ is continuous on $[a, b]$.

(ii)- $f$ is $\alpha$ differentiable on $(a, b)$.

Then, there exists $c \in(a, b)$, such that

$$
\left(T_{\alpha} f\right)(c)=\frac{f(b)-f(a)}{\left(b^{\alpha} / \alpha\right)-\left(a^{\alpha} / \alpha\right)} .
$$


Theorem 6 (see [14]). Let $a>0, \quad \alpha \in(0,1]$, and $f:[a, b] \longrightarrow R$ be a given function that satisfies

(i) $f$ is continuous on $[a, b]$.

(ii)- $f$ is $\alpha$ differentiable on $(a, b)$. $[a, b]$.

If $\left(T_{\alpha} g\right)(t)=0$ for all $t \in(a, b)$, then $f$ is a constant on

Corollary 2 (see [14]). Let $a>0, \quad \alpha \in(0,1]$, and $F, G:[a, b] \longrightarrow R$ be functions such that $\left(T_{\alpha} F\right)$ $(t)=\left(T_{\alpha} G\right)(t)$ for all $t \in(a, b)$. Then, there exists a constant $C$ such that

$$
F(t)=G(t)+C
$$

Theorem 7 Extended Mean Value Theorem (see [14]). Let $a>0, \alpha \in(0,1]$, and $f, g:[a, b] \longrightarrow R$ be functions that satisfy

(i) $f, g$ are continuous on $[a, b]$.

(ii) $f, g$ are $\alpha$ differentiable on $(a, b)$.

(iii) $\left(T_{\alpha} g\right)(t) \neq 0$ for all $t \in(a, b)$.

(iv) $g(b) \neq g(a)$.

(v) $\left(T_{\alpha} f\right)(t)$ and $\left(T_{\alpha} g\right)(t)$ not annulled simultaneously on $[a, b]$.

Then, there exists $c \in(a, b)$, such that

$$
\frac{\left(T_{\alpha} f\right)(c)}{\left(T_{\alpha} g\right)(c)}=\frac{f(b)-f(a)}{g(b)-g(a)}
$$

Remark 1. Observe that Theorem 5 is a special case of this theorem for $g(t)=\left(t^{\alpha} / \alpha\right)$.

Theorem 8 (see [14]). Let $a>0, \quad \alpha \in(0,1], \quad$ and $f:[a, b] \longrightarrow R$ be a given function that satisfies

(i) $f$ is continuous on $[a, b]$.

(ii) $f$ is $\alpha$-differentiable on $(a, b)$.

Then, we have the following:

(i) If $\left(T_{\alpha} f\right)(t)>0$ for all $t \in(a, b)$, then $f$ is increasing on $[a, b]$. (ii) If $\left(T_{\alpha} f\right)(t)<0$ for all $t \in(a, b)$, then $f$ is decreasing on $[a, b]$.

Theorem 9 (see [13]). Assume $f$ is infinitely $\alpha$ differentiable function, for some $0<\alpha \leq 1$ at the neighborhood of a point $t_{0}$. Then, $f$ has the following fractional power series expansion:

$$
f(t)=\sum_{k=0}^{\infty} \frac{\left({ }^{(k)} T_{\alpha}^{t_{0}}\right)\left(t_{0}\right)}{\alpha^{k} k !}\left(t-t_{0}\right)^{k \alpha}, \quad t_{0}<t<t_{0}+R^{(1 / \alpha)} .
$$

Here, $\left({ }^{(k)} T_{\alpha}^{t_{0}}\right)\left(t_{0}\right)$ means the application of the conformable derivative $k$ times.

Finally, the conformable partial derivative of a realvalued function with several variables is defined as follows.

Definition 3 (see $[15,16]$ ). Let $f$ be a real-valued function with $n$ variables and $\mathbf{a}=\left(a_{1}, \ldots, a_{n}\right) \in R^{n}$ be a point whose $i^{\text {th }}$ component is positive. Then, the limit can be expressed as follows

$$
\lim _{\varepsilon \longrightarrow 0} \frac{f\left(a_{1}, \ldots, a_{i}+\varepsilon a_{i}^{1-\alpha}, \ldots, a_{n}\right)-f\left(a_{1}, \ldots, a_{n}\right)}{\varepsilon},
$$

if the above limit exists, then we have the $i^{\text {th }}$ conformable partial derivative of $f$ of the order $\alpha \in(0,1]$ at a, denoted by $\left(\partial^{\alpha} / \partial x_{i}^{\alpha}\right) f(\mathbf{a})$.

\section{Main Results}

From the definitions and results on conformable derivatives of higher order, the theorems of the mean value are easily generalized which follow the same argument as in the classical calculus [42].

Theorem 10. Let $a>0, \alpha \in(0,1]$, and $f, g:[a, b] \longrightarrow R$ be functions that satisfy

(i) $-f, g \in C^{(n-1) \alpha}([a, b])$.

(ii)- $\left({ }^{n} T_{\alpha} f\right)(t)$ and $\left({ }^{n} T_{\alpha} g\right)(t)$ exist for all $t$ in $[a, b)$.

In addition, the following $n-1$ equations are assumed:

$$
\left({ }^{k} T_{\alpha} f\right)(a)[g(b)-g(a)]=\left({ }^{k} T_{\alpha} g\right)(a)[f(b)-f(a)], \quad \text { for } k=1,2, \ldots, n-1 .
$$

Then, there exists $c \in(a, b)$, such that

$$
\left({ }^{n} T_{\alpha} f\right)(c)[g(b)-g(a)]=\left({ }^{n} T_{\alpha} g\right)(c)[f(b)-f(a)] .
$$

$$
F(t)=f(t)[g(b)-g(a)]-g(t)[f(b)-f(a)], \quad \forall t \in[a, b] .
$$

Since $F$ is continuous on $[a, b], \alpha$ differentiable on $(a, b)$, and $F(a)=F(b)$, then by Theorem 4 , there exists $c_{1} \in(a, b)$ such that 


$$
\left(T_{\alpha} f\right)\left(c_{1}\right)[g(b)-g(a)]-\left(T_{\alpha} g\right)\left(c_{1}\right)[f(b)-f(a)]=0 .
$$

Let us now consider the following function:

$$
\begin{aligned}
\left(T_{\alpha} F\right)(t)= & \left(T_{\alpha} f\right)(t)[g(b)-g(a)] \\
& -\left(T_{\alpha} g\right)(t)[f(b)-f(a)], \quad \forall t \in\left[a, c_{1}\right],
\end{aligned}
$$

which is continuous on $\left[a, c_{1}\right], \alpha$ differentiable on $\left(a, c_{1}\right)$, and it is null at the extremes of interval $\left[a, c_{1}\right]$, by virtue of the above equation and hypothesis. Then, by Theorem 4 , there exists $c_{2} \in\left(a, c_{1}\right)$ such that

$$
\left({ }^{2} T_{\alpha} f\right)\left(c_{2}\right)[g(b)-g(a)]-\left({ }^{2} T_{\alpha} g\right)\left(c_{2}\right)[f(b)-f(a)]=0 .
$$

So, we reiterate this process until we obtain the following equality:

$$
\left({ }^{n-1} T_{\alpha} f\right)\left(c_{n-1}\right)[g(b)-g(a)]-\left({ }^{n-1} T_{\alpha} h\right)\left(c_{n-1}\right)[f(b)-f(a)]=0 .
$$

Then, we consider functions: ${ }^{n-1} T_{\alpha} f$ and ${ }^{n-1} T_{\alpha} g$, that are continuous on $\left[a, c_{n-1}\right]$, and $\alpha$ differentiable on $\left(a, c_{n-1}\right)$. So, by Theorem 7 , there exists $c \in\left(a, c_{n-1}\right) \subset(a, b)$ with

$$
\left({ }^{n} T_{\alpha} f\right)(c)[g(b)-g(a)]=\left({ }^{n} T_{\alpha} g\right)(c)[f(b)-f(a)] .
$$

This completes the proof of the theorem.

$$
\left({ }^{k} T_{\alpha} f\right)(a)[g(b)-g(a)]=\left({ }^{k} T_{\alpha} g\right)(a)[f(b)-f(a)], \quad \text { for } k=1,2, \ldots, n-1 .
$$

Then, there exists $c \in(a, b)$, such that

$$
\frac{\left({ }^{n} T_{\alpha} f\right)(c)}{\left({ }^{n} T_{\alpha} g\right)(c)}=\frac{f(b)-f(a)}{g(b)-g(a)} .
$$

Proof. Using the formula (21) and the fact that $\left({ }^{n} T_{\alpha} g\right)(t) \neq 0$ $\forall t \in(a, b)$, it follows that $g(b)-g(a) \neq 0$.

Dividing the two members of equality

$$
\left({ }^{n} T_{\alpha} f\right)(c)[g(b)-g(a)]=\left({ }^{n} T_{\alpha} g\right)(c)[f(b)-f(a)],
$$

by the product $\left({ }^{k} T_{\alpha} g\right)(c)[g(b)-g(a)]$, the desired result is obtained.

We end this section by obtaining the value of conformable Taylor remainder through the generalized conformable theorems of the mean value.

Definition 4. Let an open set $X \subset R, a \in X, \alpha \in(0,1]$, and $f: X \longrightarrow R$ be a function that satisfies
Remark 2. The generalized conformable formula of extended mean value theorem is derived from previous theorem by taking $g(t)=\left(t^{\alpha}-a^{\alpha}\right)^{n}$.

Theorem 11. Let $a_{0}>0, \quad a \in\left(a_{0}, b\right) \quad \alpha \in(0,1]$, and $f:\left(a_{0}, b\right] \longrightarrow R$ be a function that satisfies

(i) $f$ is continuous on $[a, b]$.

(ii)- $f$ is $n-1$ times $\alpha$ differentiable on $(a, b)$.

(iii)- $\left({ }^{n} T_{\alpha} f\right)(t)$ exist for all $t$ in $[a, b)$.

In addition, the following $n-1$ equations are assumed:

$\left(T_{\alpha} f\right)(a)=\left({ }^{2} T_{\alpha} f\right)(a)=\cdots=\left({ }^{n-1} T_{\alpha} f\right)(a)=0$.

Then, there exists $c \in(a, b)$, such that

$$
f(b)-f(a)=\frac{\left({ }^{n} T_{\alpha} f\right)(c)}{\alpha^{n} \cdot n !}\left(t^{\alpha}-a^{\alpha}\right)^{n} .
$$

Remark 3. A generalization of the conformable formula of mean value of Cauchy is also obtained.

Theorem 12. Let $a_{0}>0, \quad a \in\left(a_{0}, b\right), \quad \alpha \in(0,1]$, and $f, g:\left(a_{0}, b\right] \longrightarrow R$ be functions that satisfy

(i) $f, g$ are continuous on $[a, b]$.

(ii) $f, g$ are $n-1$ times $\alpha$ differentiable on $(a, b)$.

(iii) $\left({ }^{n} T_{\alpha} f\right)(t)$ and $\left({ }^{n} T_{\alpha} g\right)(t)$ exist for all $t$ in $[a, b)$.

(iv)- $\left({ }^{n} T_{\alpha} g\right)(t) \neq 0 \forall t \in(a, b)$.

In addition, the following $n-1$ equations are assumed:
Theorem 13. Let an open set $X \subset R, a \in X, \alpha \in(0,1]$, and $f: X \longrightarrow R$. If $f$ is $n+1$ times $\alpha$ differentiable on $[a, t] \subset X$, Then, there exists $c \in(a, t)$, such that

$$
R(t)=\left({ }^{n+1} T_{\alpha} f\right)(c) \cdot \frac{\left(t^{\alpha}-a^{\alpha}\right)^{n+1}}{\alpha^{n+1} \cdot(n+1) !},
$$

where $R$ is called the conformable Lagrange form of the remainder. 
Proof. By applying Theorem 12 to a function $R=f-p_{n}$ and using the fact that $\left({ }^{n+1} T_{\alpha} R\right)(t)=\left({ }^{n+1} T_{\alpha} f\right)(t)$ and $\left({ }^{k} T_{\alpha} R\right)(a)=0$ for $k=1,2, \ldots, n$, our result is followed.

\section{Applications to Multivariable Calculus}

In this section, we will introduce the conformable version of two interesting classical results on functions of several variables [42]. Using the conformable formula of finite increments [16], these results will be proven.

Theorem 14. Let $\alpha \in(0,1], f: X \longrightarrow R$ be a real-valued function defined in an open and convex set $X \subset R^{n}$, such that for all $\mathbf{x}=\left(x_{1}, \ldots, x_{n}\right) \in X$, each $x_{i}>0$. If the conformable partial derivative of $f$ with respect to $x_{i}$ exists and is null on $X$, then $f(\mathbf{x})=f\left(x^{\prime}\right)$ for any points $\mathbf{x}=\left(x_{1}\right.$, $\left.\ldots, x_{i}, \ldots, x_{n}\right), x^{\prime}=\left(x_{1}, \ldots, x_{i}^{\prime}, \ldots, x_{n}\right) \in X$, for $i=1,2$, $\ldots, n$.

Proof. Since $x^{\prime}$ is a convex set and $\mathbf{x}=\left(x_{1}, \ldots x_{i}, \ldots\right.$, $\left.x_{n}\right), x^{\prime}=\left(x_{1}, \ldots, x_{i}^{\prime}, \ldots, x_{n}\right) \in X$, all points of the line segment $\left[\mathbf{x}, x^{\prime}\right]$ are also in $X$, so the function $g$ is defined in the interval of endpoints $x_{i}$ and $x_{i}^{\prime}$ :

$$
t \mapsto g(t)=f\left(x_{1}, \ldots, x_{i-1}, t, x_{i+1}, \ldots, x_{n}\right) .
$$

This function is $\alpha$ differentiable on the above interval, and its derivative at a point $t$ is given by

$$
\left(T_{\alpha} g\right)(t)=\frac{\partial^{\alpha} f\left(x_{1}, \ldots, t, \ldots, x_{n}\right)}{\partial x_{i}^{\alpha}} .
$$

Therefore, by applying Theorem 5 , there is a point $c_{i}$ between $x_{i}$ and $x_{i}^{\prime}$, such that

$$
g\left(x_{i}^{\prime}\right)-g\left(x_{i}\right)=\left(\frac{x_{i}^{\prime \alpha}}{\alpha}-\frac{x_{i}^{\alpha}}{\alpha}\right) \cdot\left(T_{\alpha} g\right)\left(c_{i}\right) \text {. }
$$

Since point $\mathbf{c}=\left(x_{1}, \ldots, c_{i}, \ldots, x_{n}\right) \in X$; therefore, $\left(\partial^{\alpha} f(\mathbf{c}) / \partial x_{i}^{\alpha}\right)=0$, and the above equality leads to

$$
f\left(x^{\prime}\right)-f(\mathbf{x})=\left(x_{i}^{\prime}-x_{i}\right) \cdot \frac{\partial^{\alpha} f(c)}{\partial x_{i}^{\alpha}},
$$

then $f(\mathbf{x})=f\left(x^{\prime}\right)$, for $i=1,2, \ldots, n$, as we wanted to prove.

Finally, we introduce the first formula of finite increments for functions of several variables, involving conformable partial derivatives.

Theorem 15. Let $\mathbf{a}=\left(a_{1}, a_{2}, \ldots, a_{n}\right), \mathbf{b}=\left(b_{1}, b_{2}, \ldots, b_{n}\right) \in$ $R^{n}, \quad \mathbf{x}_{0}, \mathbf{x}_{1}, \ldots, \mathbf{x}_{\mathbf{n}}$ be points $\mathbf{x}_{\mathbf{i}}=\left(b_{1}, \ldots, b_{i}, a_{i+1}, \ldots, a_{n}\right)$ (note that $\mathbf{x}_{0}=\mathbf{a}$ and $\mathbf{x}_{\mathbf{n}}=\mathbf{b}$ ), and line segment $S_{i}=\left[\mathbf{x}_{\mathbf{i}-1}, \mathbf{x}_{\mathbf{i}}\right]$, for $i=1,2, \ldots, n$. Let $\alpha \in(0,1]$, and $f: X \longrightarrow R$ be a realvalued function defined in an open set $X \subset R^{n}$ containing line segments $S_{1}, S_{2}, \ldots, S_{n}$, such that for all $\mathbf{x}=\left(x_{1}, \ldots, x_{n}\right) \in X$, each $x_{i}>0$. If the conformable partial derivative of $f$ with respect to $x_{i}$ exists on $X$, then there is a point $c_{i}$ between $a_{i}$ and $b_{i}$, for $i=1,2, \ldots, n$, such that

$$
f\left(b_{1}, b_{2}, \ldots b_{n}\right)-f\left(a_{1}, a_{2}, \ldots, a_{n}\right)=\sum_{i=1}^{n}\left(\frac{b_{i}^{\alpha}}{\alpha}-\frac{a_{i}^{\alpha}}{\alpha}\right) \cdot \frac{\partial^{\alpha} f\left(b_{1}, \ldots, b_{i-1}, c_{i}, a_{i+1} \ldots, a_{n}\right)}{\partial x_{i}^{\alpha}} .
$$

Proof. First, we will express the difference $f(\mathbf{b})-f(\mathbf{a})$ as follows:

$$
f(\mathbf{b})-f(\mathbf{a})=f\left(\mathbf{x}_{\mathbf{n}}\right)-f\left(\mathbf{x}_{0}\right)=\sum_{i=1}^{n}\left[f\left(\mathbf{x}_{\mathbf{i}}\right)-f\left(\mathbf{x}_{\mathbf{i}-1}\right)\right] .
$$

Let us now consider, for $i=1,2, \ldots, n$, the real function $g_{i}$ of the real variable $t$, defined on the closed interval of endpoints $a_{i}$ and $b_{i}$, by

$$
t \longmapsto g_{i}(t)=f\left(b_{1}, \ldots, b_{i-1}, t, a_{i+1}, \ldots, a_{n}\right) .
$$

Since the conformable partial derivative of $f$ with respect to $x_{i}$ exists on $X$ and $S_{i} \subset X$, then $g_{i}$ is $\alpha$ differentiable on the above interval, and its derivative at a point $t$, is given by

$$
\left(T_{\alpha} g_{i}\right)(t)=\frac{\partial^{\alpha} f\left(b_{1}, \ldots, b_{i-1}, t, a_{i+1}, \ldots, a_{n}\right)}{\partial x_{i}^{\alpha}} .
$$

Therefore, by applying Theorem 5 , there is a point $c_{i}$ between $a_{i}$ and $b_{i}$, such that

$$
g_{i}\left(b_{i}\right)-g_{i}\left(a_{i}\right)=\left(\frac{b_{i}^{\alpha}}{\alpha}-\frac{a_{i}^{\alpha}}{\alpha}\right) \cdot\left(T_{\alpha} g_{i}\right)\left(c_{i}\right) .
$$

Then, it is verified that

$$
f\left(\mathbf{x}_{\mathbf{i}}\right)-f\left(\mathbf{x}_{\mathbf{i}-1}\right)=\left(\frac{b_{i}^{\alpha}}{\alpha}-\frac{a_{i}^{\alpha}}{\alpha}\right) \cdot \frac{\partial^{\alpha} f\left(b_{1}, \ldots, b_{i-1}, t, a_{i+1}, \ldots, a_{n}\right)}{\partial x_{i}^{\alpha}} .
$$

By taking the above expression to equation (32), our result is followed.

\section{Conclusion}

In this research work, some new results regarding the conformable mean value theorems have been proposed. As in classical calculus, higher-order derivatives have been applied to generalize the mean value theorems. Likewise, the Lagrange expression has been established for the Taylor conformable remainder. In the context of the calculus of functions of several variables, according to the conformable mean value theorem, the functions in which one of its conformable partial derivatives is null have been characterized, and the first conformable formula 
of finite increments has been obtained. The findings of this investigation indicate that the results obtained in the sense of the conformable derivative coincide with the results obtained in the classical case of integer order. Finally, our obtained results, in addition to a theoretical interest, show great potential to be applied in a future research work concerning various applications in the field of natural sciences and engineering.

\section{Data Availability}

No data were used to support this study.

\section{Conflicts of Interest}

The authors declare that there are no conflicts of interest regarding the publication of this paper.

\section{References}

[1] A. Kilbas, H. Srivastava, and J. Trujillo, Theory and Applications of Fractional Differential Equations, North-Holland, Amsterdam, Netherlands, 2006.

[2] K. S. Miller, An Introduction to Fractional Calculus and Fractional Differential Equations, John Wiley and Sons, Hoboken, NJ, USA, 1993.

[3] M. M. Matar, M. I. Abbas, J. Alzabut, M. K. A. Kaabar, S. Etemad, and S. Rezapour, "Investigation of the p-laplacian nonperiodic nonlinear boundary value problem via generalized Caputo fractional derivatives," Advances in Difference Equations, vol. 2021, no. 68, pp. 1-18, 2021.

[4] S. Rezapour, A. Imran, A. Hussain, F. Martínez, S. Etemad, and M. K. A. Kaabar, "Condensing functions and approximate endpoint criterion for the existence analysis of quantum integrodifference FBVPs," Symmetry, vol. 13, no. 3, p. 469, 2021.

[5] F. Martínez, I. Martínez, M. K. A. Kaabar, R. Ortíz-Munuera, and S. Paredes, "Note on the conformable fractional derivatives and integrals of complex-valued functions of a real variable," IAENG International Journal of Applied Mathematics, vol. 50, no. 3, pp. 609-615, 2020.

[6] R. Khalil, M. Al Horani, A. Yousef, and M. Sababheh, "A new definition of fractional derivative," Journal of Computational and Applied Mathematics, vol. 264, pp. 65-70, 2014.

[7] M. Kaabar, "Novel methods for solving the conformable wave equation," Journal of New Theory, vol. 31, pp. 56-85, 2020.

[8] R. Almeida, M. Guzowska, and T. Odzijewicz, "A remark on local fractional calculus and ordinary derivatives," Open Mathematics, vol. 14, no. 1, pp. 1122-1124, 2016.

[9] D. Zhao and M. Luo, "General conformable fractional derivative and its physical interpretation," Calcolo, vol. 54, no. 3 , pp. 903-917, 2017.

[10] R. Khalil, M. Al Horani, and M. Abu Hammad, "Geometric meaning of conformable derivative via fractional cords," Journal of Mathematics and Computer Science, vol. 19, no. 4, pp. 241-245, 2019.

[11] N. H. Tuan, T. B. Ngoc, D. Baleanu, and D. O’Regan, “On well-posedness of the sub-diffusion equation with conformable derivative model," Communications in Nonlinear Science and Numerical Simulation, vol. 89, Article ID 105332, 2020.

[12] F. Martínez, I. Martínez, M. K. A. Kaabar, and S. Paredes, "On conformable laplace's equation," Mathematical Problems in Engineering, vol. 2021, Article ID 5514535, 10 pages, 2021.
[13] T. Abdeljawad, "On conformable fractional calculus," Journal of Computational and Applied Mathematics, vol. 279, pp. 57-66, 2015.

[14] O. S. Iyiola and E. R. Nwaeze, "Some new results on the new conformable fractional calculus with application using D 'Alambert approach," Progress in Fractional Differentiation and Applications, vol. 2, no. 2, pp. 115-122, 2016.

[15] A. Atangana, D. Baleanu, and A. Alsaedi, "New properties of conformable derivative," Open Mathematics, vol. 13, pp. 5763, 2015.

[16] N. Yazici and U. Gözütok, "Multivariable conformable fractional calculus," Filomat, vol. 32, no. 2, pp. 45-53, 2018.

[17] F. Martínez, I. Martínez, and S. Paredes, "Conformable Euler 's theorem on homogeneous functions," Computational Mathematical Methods, vol. 1, no. 5, pp. 1-11, 2018.

[18] E. Ünal, A. An, and E. Çelik, "Solutions of sequential conformable fractional differential equations around an ordinary point and conformable fractional hermite differential equation," British Journal of Applied Science \& Technology, vol. 10, no. 2, pp. 1-11, 2015.

[19] M. Al Masalmeh, "Series method to solve conformable fractional ric-cati differential equations," International Journal of Applied Mathematical Research, vol. 6, no. 1, pp. 30-33, 2017.

[20] M. Al Horani and R. Khalil, "Total fractional differential with applications to exact fractional differential equations," International Journal of Computer Mathematics, vol. 95, no. 6-7, pp. 1444-1452, 2018.

[21] M. Al Horani, M. Abu Hammad, and R. Khalil, "Variation of parameters for local fractional nonhomogenous linear differential equations," Journal of Mathematics and Computer Science, vol. 16, no. 2, pp. 147-153, 2016.

[22] R. Khalil, M. Al Horani, and D. Anderson, "Undetermined coefficients for local fractional differential equations," Journal of Mathematics and Computer Science, vol. 16, no. 2, pp. 140-146, 2016.

[23] M. A. Hammad and R. Khalil, "Abel's formula and wronskian for conformable fractional differential equations," International Journal of Differential Equations and Applications, vol. 13, no. 2, pp. 177-183, 2014.

[24] M. A. Hammad and R. Khalil, "Legendre fractional differential equation and Legendre fractional polynomials," International Journal of Applied Mathematical Research, vol. 3, no. 3, pp. 214-219, 2014.

[25] F. S. Silva, M. D. Moreira, and M. A. Moret, "Conformable laplace transform of fractional differential equations," $A x$ ioms, vol. 7, no. 55, 2018.

[26] Z. Al-Zhour, N. Al-Mutairi, F. Alrawajeh, and R. Alkhasawneh, "Series solutions for the laguerre and laneemden fractional differential equations in the sense of conformable fractional derivative," Alexandria Engineering Journal, vol. 58, no. 4, pp. 1413-1420, 2019.

[27] M. A. Hammad, H. Alzaareer, H. Al-Zoubi, and H. Dutta, "Fractional Gauss hypergeometric differential equation," Journal of Interdisciplinary Mathematics, vol. 22, no. 7, pp. 1113-1121, 2019.

[28] F. Martínez, I. Martínez, and S. Paredes, "A note on integrating factors of a conformable fractional differential equation," International Journal of Applied Physics and Mathematics, vol. 10, no. 4, pp. 143-152, 2020.

[29] F. Martínez, I. Martínez, M. K. A. Kaabar, and S. Paredes, "Some new results on conformable fractional power series," Asia Pacific Journal of Mathematics, vol. 7, no. 3, pp. 1-14, 2020. 
[30] I. Abu Hammad and R. Khalil, "Fractional fourier series with applications," American Journal of Computational and Applied Mathematics, vol. 4, no. 6, pp. 187-191, 2014.

[31] I. Abu Hammad and R. Khalil, "Some new results on conformable fractional differential equations," International Journal of Pure and Applied Mathematics, vol. 94, no. 2, pp. 215-314, 2020.

[32] R. Khalil and H. Abu-Shaab, "Solution of some conformable fractional power series," Asia Pacific Journal of Mathematics, vol. 103, no. 4, pp. 667-673, 2015.

[33] F. Martínez, I. Martínez, I. Martínez, M. K. A. Kaabar, and S. Paredes, "New results on complex conformable integral," AIMS Mathematics, vol. 5, no. 6, pp. 7695-7710, 2020.

[34] Z. Korpinar, A. S. Alshomrani, M. Inc, A. S. Alshomrani, and D. Baleanu, "The deterministic and stochastic solutions of the Schrodinger equation with time conformable derivative in birefrigent fibers," AIMS Mathematics, vol. 5, no. 3, pp. 2326-2345, 2020.

[35] Z. Korpinar, A. S. Alshomrani, and D. Baleanu, "On exact special solutions for the stochastic regularized long waveBurgers equation," Advances in Difference Equations, vol. 2020, no. 1, p. 433, 2020.

[36] M. Kaplan, "Applications of two reliable methods for solving a nonlinear conformable time-fractional equation," Optical and Quantum Electronics, vol. 49, no. 9, p. 312, 2017.

[37] M. Kaplan and A. Akbulut, "Application of two different algorithms to the approximate long water wave equation with conformable fractional derivative," Arab Journal of Basic and Applied Sciences, vol. 25, no. 2, pp. 77-84, 2018.

[38] M. M. A. Khater, Y.-M. Chu, R. A. M. Attia, M. Inc, and D. Lu, "On the analytical and numerical solutions in the quantum magnetoplasmas: the atangana conformable derivative $(1+3)$ ZK equation with power-law nonlinearity," Advances in Mathematical Physics, vol. 2020, Article ID 5809289, 10 pages, 2020.

[39] A. Akbulut and M. Kaplan, "Auxiliary equation method for time-fractional differential equations with conformable derivative," Computers \& Mathematics with Applications, vol. 75, no. 3, pp. 876-882, 2018.

[40] D. Kumar and M. Kaplan, "New analytical solutions of $(2+1)-$ dimensional conformable time fractional Zoomeron equation via two distinct techniques," Chinese Journal of Physics, vol. 56, no. 5, pp. 2173-2185, 2018.

[41] D. Kumar, M. Kaplan, M. Haque, M. S. Osman, and D. Baleanu, "A variety of novel exact solutions for different models with the conformable derivative in shallow water," Frontiers in Physics, vol. 8, no. 177, pp. 1-13, 2020.

[42] M. Spivak, Calculus, W.A. Benjamin, Inc., Menlo Park, CA, USA, 2nd edition, 1988. 